\title{
EFECTO DE FUNGICIDAS SOBRE CARACTERES AGRONÓMICOS, RENDIMIENTO Y TIZONES FOLIARES EN TRIGO DE TEMPORAL EN MÉXICO
}

\author{
EFFECT OF FUNGICIDES ON AGRONOMIC TRAITS, YIELD AND \\ LEAF BLIGHTS IN RAINFED WHEAT IN MEXICO
}

\author{
Luis A. Mariscal-Amaro'*, Héctor E. Villaseñor-Mir², \\ Ernesto Solís-Moya', René Hortelano-Santa Rosa ${ }^{2}$ y Eliel Martínez-Cruz ${ }^{2}$
}

\begin{abstract}
${ }^{1}$ Instituto Nacional de Investigaciones Forestales, Agrícolas y Pecuarias (INIFAP), Campo Experimental Bajío, Celaya, Guanajuato, México. ${ }^{2}$ INIFAP, Campo Experimental Valle de México, Coatlinchán, Texcoco, Estado de México, México.
\end{abstract}

*Autor de correspondencia (mariscal.luis@inifap.gob.mx)

\section{RESUMEN}

El trigo (Triticum aestivum L.) sembrado bajo condiciones de temporal en México hospeda hongos causantes de manchas y tizones foliares que reducen el rendimiento hasta en $60 \%$. Ante la falta de materiales resistentes a estas enfermedades y las pérdidas de rendimiento que ocasionan en las variedades actuales sembradas bajo estas condiciones se recurre al control químico como alternativa complementaria en este cereal. Actualmente existe poca información del efecto de fungicidas con diferentes ingredientes activos sobre la severidad de estas enfermedades y otros rasgos agronómicos. El objetivo de este estudio fue probar la efectividad de fungicidas foliares sobre la severidad de la enfermedad medida como el área foliar dañada y observar su efecto sobre los caracteres agronómicos y de rendimiento. El estudio se llevó a cabo en tres localidades de Valles Altos del centro de México, donde se probaron ocho fungicidas sistémicos y tres de contacto. Los fungicidas sólo tuvieron efecto altamente significativo en los días a madurez, donde la azoxistrobina + propiconazole retrasó la madurez de la planta hasta en $8 \mathrm{~d}$ comparado con el testigo. Todos los fungicidas redujeron el área foliar dañada hasta en $63.3 \%$ y aumentaron el rendimiento de grano hasta en $56 \%$. Los fungicidas a base de azoxistrobina y los de la familia de los triazoles indujeron el efecto stay-green (demora en la senescencia foliar) y mejoraron la sanidad de las plantas. Se observó una correlación positiva entre días a madurez y rendimiento de grano, y negativa entre el porcentaje de área foliar dañada y rendimiento de grano. La aplicación de fungicidas foliares para el manejo de manchas y tizones foliares en trigo se recomienda como una alternativa para proteger el cultivo y aumentar el rendimiento en trigo.

Palabras clave: Triticum aestivum, Septoria tritici Desm., fungicidas, rendimiento, tizones foliares.

\section{SUMMARY}

Wheat (Triticum aestivum L.) planted under rainfed conditions hosts fungi that cause spots and leaf blight that reduce yield by up to $60 \%$. Given the lack of varieties resistant to these diseases and the yield losses that they cause in the current varieties planted under these conditions, chemical control is used as a complementary alternative in this cereal. Currently, there is little information on the effect of fungicides with different active ingredients on the severity of these diseases and other agronomic traits. The objective of this study was to test the effectiveness of foliar fungicides on the severity of the disease measured as the damaged leaf area and to observe their effect on agronomic traits and yield. The study was carried out at three locations of the highlands of central Mexico, where eight systemic and three contact fungicides were tested. Fungicides only had a highly significant effect on days to maturity where azoxystrobin + propiconazole delayed plant maturity by up to $8 \mathrm{~d}$ compared to the control. All fungicides reduced the damaged leaf area by up to $63.3 \%$ and increase grain yield by up to $56 \%$. Azoxystrobin-based fungicides and those of the triazole family induced the stay-green (delayed foliar senescence) effect and improved plant health. A positive correlation was observed between days to maturity and grain yield, and anegative one between the percentage of damaged leaf area and grain yield. The application of foliar fungicides for the management of leaf spots and blights on wheat is recommended as an alternative to protect the crop and increase the yield in wheat.

Index words: Triticum aestivum, Septoria tritici Desm., fungicides, leaf blights, yield.

\section{INTRODUCCIÓN}

El trigo (Triticum aestivum L.) es el cereal más utilizado en la alimentación humana debido a su alto valor nutritivo (Noriega-Carmona et al., 2019); en México se encuentra en segundo lugar de producción, solamente después del maíz (Zea mays L.). En 2018 se sembraron 543,473 ha con una producción estimada de 2,943,445 t y un rendimiento de $5.44 \mathrm{t} \mathrm{ha}^{-1}$ (SIAP, 2019).

La roya de la hoja (Puccinia triticina E.) y la roya lineal amarilla (Puccinia striiformis f. sp. tritici) son los principales problemas fitosanitarios del trigo (Díaz et al., 2018). En siembras bajo condiciones de temporal también se presentan hongos causantes de manchas y tizones foliares, de los cuales se han reportado especies como Helminthosporium spp., Bipolaris spp. (Bautista et al., 2011; Mariscal-Amaro et al., 2017), además de Septoria tritici en regiones productoras de trigo de riego en México (Mariscal et al., 2017) y los géneros Alternaria, Fusarium y Curvularia (Mariscal, 2014; Mariscal-Amaro et al., 2017).

La especie Bipolaris sorokiniana (sin. H. sativum) causa pérdidas de rendimiento del 20 al 60 \% (Acharya et al., 2011; 
Duveiller et al., 2007; Rattu et al., 2011) mientras que con S. tritici las pérdidas son del $44 \%$ (Rodríguez-Contreras et al., 2008). Estos hongos, además de reducir el rendimiento, contaminan la semilla de trigo y ocasionan el manchado de grano, pérdidas en almacén, pudriciones de raíz y tallo, reducción de la germinación y muerte de plántulas cuando el grano se usa para siembra (Mariscal-Amaro et al., 2017). Actualmente, las variedades mexicanas de trigo que se siembran bajo temporal poseen tolerancia o moderada susceptibilidad a manchas y tizones foliares y presentan pérdidas de rendimiento de hasta $36 \%$ (Villaseñor et al., 2018), por lo que se recurre al control químico como alternativa complementaria para evitar estas pérdidas. Existe en México poca información sobre el efecto de fungicidas con diferentes ingredientes activos sobre la reducción de la severidad de manchas y tizones foliares y sobre caracteres agronómicos y de rendimiento. Por lo anterior, el presente estudio se llevó a cabo con el objetivo de probar la efectividad de fungicidas sistémicos y de contacto sobre estas enfermedades en trigo sembrado bajo temporal y su efecto sobre el rendimiento y otras características agronómicas. La hipótesis planteada fue que al menos uno de los fungicidas reduce significativamente la severidad de estas enfermedades, tiene efecto sobre alguno de los caracteres agronómicos y favorece el aumento del rendimiento en el cultivo.

\section{MATERIALES Y MÉTODOS}

\section{Área de estudio}

El experimento se llevó a cabo en el ciclo primaveraverano 2016 bajo condiciones de temporal en tres parcelas experimentales del Instituto Nacional de Investigaciones Forestales, Agrícolas y Pecuarias (INIFAP), Campo Experimental Valle de México en las localidades de Juchitepec (190 08' 7.5" N, $98^{\circ} 52^{\prime} 7.5^{\prime \prime} \mathrm{O}$, $2443 \mathrm{msnm}$,), clima templado subhúmedo con lluvias en verano, con una precipitación media anual de $950 \mathrm{~mm}$ y temperatura media anual de $14.4^{\circ} \mathrm{C}$; Santa Lucía (19² $26^{\prime}$ 45.02" N, $98^{\circ}$ 53' 36.64" O, 2438 msnm), clima templado subhúmedo con lluvias en verano, con una precipitación media anual de $600 \mathrm{~mm}$ y temperatura media anual de $16.4^{\circ} \mathrm{C}$, y Nanacamilpa (19² 29' 20.22" N, $98^{\circ} 45^{\prime \prime}$ O, 2581 msnm), clima templado subhúmedo con lluvias en verano, precipitación media anual de $750 \mathrm{~mm}$ y temperatura media anual de $11.1^{\circ} \mathrm{C}$ (CONAGUA, 2019; INEGI, 2019). Las dos primeras localidades pertenecen al Estado de México y la tercera al estado de Tlaxcala.

\section{Tratamientos, diseño experimental y manejo agronómico}

Se evaluaron tres fungicidas de contacto y ocho sistémicos (Cuadro 1). Se usaron las dosis recomendadas por el fabricante. Se realizó una sola aspersión de los fungicidas cuando se presentó de 10 a 20\% deespigamiento, lo que coincidió con los primeros síntomas de manchas y tizones foliares, cuyos hongos causantes se presentaron de forma natural y fueron identificados posteriormente. En el tratamiento testigo sólo se asperjó agua. Los tratamientos se distribuyeron bajo un diseño de bloques completos al azar con cuatro repeticiones, la unidad experimental fue de $3.6 \mathrm{~m}^{2}$. En las tres parcelas se sembró la variedad de trigo Náhuatl F2000, que es de moderadamente susceptible a susceptible a enfermedades foliares causadas por $B$. sorokiniana, S. tritici y Fusarium spp. (Orantes-García et al., 2011; Villaseñor-Mir et al., 2005).

La fertilización, control de malezas y otras labores culturales se realizaron siguiendo las recomendaciones del Programa de Mejoramiento Genético de Trigo de Temporal del INIFAP (Hortelano et al., 2013).

\section{Variables evaluadas}

Las variables evaluadas fueron días a floración (DF), días a madurez (DM), altura de planta (AP) en $\mathrm{cm}$, rendimiento de grano $(R G)$ en $\mathrm{kg} \mathrm{ha}^{-1}$ y severidad de las enfermedades medida como el porcentaje de área foliar dañada (AFD). Para la lectura de las manchas y tizones foliares se utilizó la escala de dos dígitos (Saari y Prescott, 1975) y se transformó a porcentaje del AFD mediante la fórmula siguiente propuesta por Duveiller et al. (2005):

$$
\% \mathrm{AFD}=(\mathrm{D} 1 / 9) \times(\mathrm{D} 2 / 9) \times 100
$$

donde: la incidencia D1 (primer dígito) indica el progreso de la enfermedad en la altura de la planta y la severidad D2 (segundo dígito) el área foliar con síntomas de la enfermedad.

Las lecturas se hicieron en la etapa de grano masoso (Rodríguez-Contreras et al., 2008), cuando aún había follaje verde en la planta.

Para la identificación de los hongos causantes de las enfermedades se realizó un muestreo directo en las parcelas testigo de cada localidad. De cada parcela se eligieron cuatro plantas con síntomas de tizones o 
Cuadro 1. Ingredientes activos de los fungicidas utilizados en el experimento, modo de acción y dosis aplicada.

\begin{tabular}{llcc}
\hline No. & Ingrediente activo & Modo de acción & Dosis ha $^{-1}$ \\
\hline 1 & Captán $(50 \%)^{+}$ & Contacto & $300 \mathrm{~g}$ \\
2 & Clorotalonil $(54 \%)$ & Contacto & $2.5 \mathrm{~L}$ \\
3 & Mancozeb $(80 \%)$ & Contacto & $2.0 \mathrm{~kg}$ \\
4 & Benomilo $(50 \%)$ & Sistémico & $60 \mathrm{~g}$ \\
5 & Tebuconazole $(25 \%)$ & Sistémico & $0.5 \mathrm{~L}$ \\
6 & Metalaxil-M $(4 \%)+$ clorotalonil $(40 \%)$ & Sistémico & $3.5 \mathrm{~L}$ \\
7 & Azoxistrobina $(20 \%)+$ difenoconazole $(12.5 \%)$ & Sistémico & $100 \mathrm{~mL}$ \\
8 & Tiofanato metílico (70\%) & Sistémico & $1.0 \mathrm{~kg}$ \\
9 & Propiconazole $(25.5 \%)$ & Sistémico & $0.5 \mathrm{~L}$ \\
10 & Procloraz $(42.10 \%)$ & Sistémico & $1.5 \mathrm{~L}$ \\
11 & Azoxistrobina $(7.5 \%)+$ propiconazole $(12.5 \%)$ & Sistémico & $1.0 \mathrm{~L}$ \\
\hline
\end{tabular}

${ }^{\dagger}$ Entre paréntesis el porcentaje de ingrediente activo del fungicida por $\mathrm{kg} \circ \mathrm{L}$ de producto comercial.

manchas foliares, y de ellas se seleccionó una hoja del tercio inferior con los síntomas por planta. Esto se realizó en las cuatro repeticiones ( 16 hojas en total) en todas las localidades (48 hojas en total). Las hojas se almacenaron en bolsas glassine para su preservación, se prensaron y secaron para evitar que se fragmentaran (Zillinsky, 1984). En el laboratorio, de cada hoja bandera se cortó una porción central de $8 \mathrm{~cm}$ de longitud, se desinfectó por 90 s con hipoclorito de sodio $5 \%$. Las porciones se lavaron tres veces con agua destilada estéril y se secaron en papel absorbente estéril. Dos porciones de hoja se colocaron en cámara húmeda (cajas de Petri con $\varnothing=10 \mathrm{~cm}$ ) con papel filtro absorbente estéril humedecido con agua destilada estéril. Las cámaras se sellaron con parafilm, se etiquetaron y mantuvieron a $24^{\circ} \mathrm{C}$ por $48 \mathrm{~h}$ y se revisaron; posteriormente, los intervalos de revisión fueron cada 24 h, según la tasa de crecimiento de los hongos. La identificación se realizó mediante observación directa de las características de las colonias y de la morfología de los conidios en preparaciones temporales con lactofenol $10 \%$ con ayuda de un microscopio compuesto. La identificación morfológica se realizó mediante los descriptores utilizados por Leslie y Summerell (2006) y Zillinsky (1984).

\section{Análisis estadístico}

Se realizó análisis de varianza mediante modelos lineales generalizados (GLM) de un diseño de bloques completos al azar y prueba de medias de Tukey $(P \leq 0.05)$, además de un análisis de correlación y regresión entre las variables utilizando el programa estadístico SAS® versión 9.3 (SAS Institute, 2011).

\section{RESULTADOS Y DISCUSIÓN}

\section{Incidencia de enfermedades}

En la localidad de Juchitepec se identificaron los hongos S. tritici (sin. Zymoseptoria tritici), Pyrenophora triticirepentis, Cochliobolus sativus y Fusarium spp.; en Santa Lucía se presentaron P. tritici-repentis, S. tritici y Fusarium spp., mientras que en Nanacamilpa se identificaron $S$. tritici, S. nodorum, P. tritici-repentis, C. sativus, Alternaria spp. y Fusarium spp. En las parcelas de los tratamientos testigo se presentó desde 70 hasta $100 \%$ de AFD.

\section{Efecto de tratamientos sobre variables agronómicas y fitopatológicas}

En el Cuadro 2 se presenta la comparación de medias de las variables agronómicas y fitopatológicas por localidad. Se observó que en las localidades de Juchitepec y Nanacamilpa, donde se han registrado las mayores precipitaciones durante los meses del ciclo del cultivo (junionoviembre), hasta $623 \mathrm{~mm}$ y $554 \mathrm{~mm}$, respectivamente (CONAGUA, 2019), se obtuvieron los mayores RG, hasta 60 \% más en comparación con la localidad de Santa Lucía (494 mm); así mismo, se incrementaron las variables DF $\mathrm{DM}, \mathrm{AP}$ y $\mathrm{AFD}$, esta última con un aumento del $27 \%$. Esta tendencia coincide con lo reportado por otros autores, en el sentido que en las localidades o años con mayores precipitaciones, como Juchitepec con $853 \mathrm{~mm}$ anuales (Rodríguez-Contreras et al., 2008) o Nanacamilpa con 640 mm (Villaseñor et al., 2012), se han obtenido rendimientos de grano mayores hasta en $18 \%$. 
Cuadro 2. Comparación de medias de las variables agronómicas y fitopatológicas en las localidades de evaluación. Ciclo primavera-verano 2016.

\begin{tabular}{lccccc}
\hline Localidad & DF & DM & AP $(\mathrm{cm})$ & AFD (\%) & RG $\left(\mathrm{kg} \mathrm{ha}^{-1}\right)$ \\
\hline Santa Lucía & $60 \mathrm{c}$ & $107 \mathrm{c}$ & $84 \mathrm{~b}$ & $56 \mathrm{~b}$ & $2751 \mathrm{c}$ \\
Juchitepec & $65 \mathrm{~b}$ & $127 \mathrm{~b}$ & $95 \mathrm{a}$ & $70 \mathrm{a}$ & $4412 \mathrm{a}$ \\
Nanacamilpa & $67 \mathrm{a}$ & $135 \mathrm{a}$ & $94 \mathrm{a}$ & $71 \mathrm{a}$ & $3241 \mathrm{~b}$ \\
CV $(\%)$ & 1.10 & 0.74 & 4.26 & 8.12 & 14.37 \\
DSH (0.05) & 0.34 & 0.44 & 1.88 & 2.6 & 242 \\
\hline
\end{tabular}

Medias con letras iquales en las columnas no son estadísticamente diferentes (Tukey, P $\leq 0.05$ ). DF: días a floración, DM: días a madurez, AP. altura de planta, AFD: área foliar dañada, RG: rendimiento de grano, CV: coeficiente de variación, DSH: diferencia significativa honesta.

Se observó un aumento en la severidad de manchas y tizones foliares causados por S. tritici y P. tritici-repentis medidas a través del área bajo la curva del progreso de la enfermedad (ABCPE). Para estos dos hongos, las ABCPE tuvieron, respectivamente, valores de 3231 y 537 en los años con mayor precipitación, comparados con valores de 1699 y 370 en los años menos lluviosos (Castro et al., 2018). Gill et al. (2015), al evaluar la relación entre el contraste climático de dos regiones de la India productoras de trigo y sus parámetros agronómicos y de rendimiento observaron una mayor altura de planta (hasta $3 \mathrm{~cm}$ más) y un incremento del rendimiento (hasta $14 \%$ ) conforme se incrementaron los niveles de riego en el cultivo. Wegulo et al. (2012) mencionan que una mayor cantidad de lluvia tiene un impacto favorable durante la etapa de llenado de grano, lo que se refleja en el aumento del rendimiento. En el estudio de estos autores, en el año con mayor precipitación (2007 con 306 mm) comparado con 2006 (con $156 \mathrm{~mm}$ ), el rendimiento se incrementó en $1369 \mathrm{~kg} \mathrm{ha}^{-1}$ en 2007 mientras que en 2006 éste fue de $394 \mathrm{~kg} \mathrm{ha}^{-1}$. Rodríguez-Contreras et al. (2008) y Wegulo et al. (2012) mencionan que la mayor severidad de la enfermedad, en este caso un mayor porcentaje de AFD, en las localidades y años con mayor precipitación se debe a que los hongos tuvieron mejores condiciones ambientales (agua disponible sobre la lámina de las hojas) para su desarrollo, reproducción y sobrevivencia, y en sus primeras etapas, para la germinación de sus esporas, crecimiento del tubo germinativo y penetración en la planta (Aung et al., 2018).

Entre fungicidas se observaron diferencias altamente significativas para DM, RG y AFD (Cuadro 3), pero no hubo efecto sobre las variables DF y AP. Para DM, la aplicación de azoxistrobina + propiconazole provocó un retraso en la madurez de la planta con 127 d, comparados con los 119 d del testigo; así mismo, Castro et al. (2018) y Zhang et al. (2010), al aplicar estrobirulinas (familia de la azoxistrobina) y triazoles (familia del propiconazole) de forma foliar en trigo, observaron que estos fungicidas mantuvieron el área foliar verde por más tiempo lo que retrasó la madurez. Zhang et al. (2010) observaron que en las plantas tratadas con azoxistrobina se incrementaron los contenidos de clorofila y de proteína soluble y se observó mayor actividad de las enzimas antioxidantes superóxido dismutasa, catalasa y peroxidasa en la hoja bandera, por lo que sugirieron que este retraso en la senescencia inducido por el fungicida se debe a una mayor actividad enzimática antioxidante que protege a las plantas de las especies dañinas de oxígeno activo.

Se registró una reducción en el AFD en comparación con el testigo, lo que estuvo asociado con un incremento en el rendimiento (Figura 1). El mayor rendimiento de grano se registró en los tratamientos con azoxistrobina + propiconazole (5152 kg ha-1), tebuconazole (4668 kg $\left.\mathrm{ha}^{-1}\right)$, propiconazole $\left(4657 \mathrm{~kg} \mathrm{ha}^{-1}\right)$ y azoxistrobina + difenoconazole (4182 $\mathrm{kg} \mathrm{ha}^{-1}$ ), los cuales registraron ganancias en rendimiento de 156, 131, 131 y $108 \%$, respectivamente, comparados con el testigo $\left(2013 \mathrm{~kg} \mathrm{ha}^{-1}\right)$. Zhang et al. (2010) mencionaron que en trigo la aplicación de fungicidas a base de azoxistrobina o de algún triazol, ya sea tebuconazole o propiconazole, induce el efecto staygreen en el follaje, la hoja permanece más tiempo verde, y por consiguiente, hay un retraso en la senescencia de la planta (Brinkman et al., 2014), lo que permite mayor periodo de área foliar fotosintéticamente activa y mayor cantidad de asimilados disponibles para el llenado de grano, que a su vez se refleja en un mayor peso y rendimiento. Estos rendimientos se relacionaron con la severidad de las enfermedades ya que con estos fungicidas se registró la menor AFD, azoxistrobina + propiconazole con $31.7 \%$, azoxistrobina + difenoconazole $42.5 \%$, tebuconazole 44.2 $\%$ y propiconazole $47.9 \%$ (Cuadro 3, Figura 1). En general, con todos los tratamientos se obtuvo un intervalo de 5.0 a $63.3 \%$ de reducción del AFD. La menor AFD registrada por azoxistrobina + propiconazole se debe a que los dos ingredientes activos de este fungicida tienen actividad contra un amplio espectro de hongos del follaje en trigo (Beck et al., 2002; PLM Editores, 2011), además de su efecto sobre la inducción de resistencia de la planta mencionado por Zhang et al. (2010). 
Cuadro 3. Comparación de medias de las variables agronómicas y fitopatológicas en función de los fungicidas evaluados. Ciclo primavera-verano 2016.

\begin{tabular}{|c|c|c|c|c|c|}
\hline Tratamiento & DF & DM & $\mathrm{AP}(\mathrm{cm})$ & AFD (\%) & $\mathrm{RG}\left(\mathrm{kg} \mathrm{ha}^{-1}\right)$ \\
\hline Captán & $65 \mathrm{a}$ & $121 \mathrm{~d}$ & $91.1 \mathrm{a}$ & $90.0 \mathrm{a}$ & 2306 ef \\
\hline Clorotalonil & $64 \mathrm{a}$ & $122 \mathrm{~cd}$ & $90.7 \mathrm{a}$ & $80.0 \mathrm{~b}$ & 2601 def \\
\hline Mancozeb & $64 a$ & $121 \mathrm{~cd}$ & $92.3 \mathrm{a}$ & 77.5 bc & $3149 d$ \\
\hline Benomilo & $64 \mathrm{a}$ & $122 \mathrm{~cd}$ & $92.4 \mathrm{a}$ & 74.2 bc & 2851 de \\
\hline Tebuconazole & $65 a$ & $125 b$ & $91.6 \mathrm{a}$ & $44.2 \mathrm{e}$ & $4668 a b$ \\
\hline Metalaxil-M + clorotalonil & $64 \mathrm{a}$ & $122 \mathrm{~cd}$ & 90.8 a & $79.2 b$ & $3180 d$ \\
\hline Azoxistrobina + difenoconazole & $64 \mathrm{a}$ & $125 b$ & $89.8 \mathrm{a}$ & $42.5 \mathrm{e}$ & 4182 bc \\
\hline Tiofanato metílico & $64 \mathrm{a}$ & $122 \mathrm{c}$ & $90.5 \mathrm{a}$ & $71.7 \mathrm{c}$ & 2896 de \\
\hline Propiconazole & $64 \mathrm{a}$ & $124 b$ & $89.4 \mathrm{a}$ & 47.9 e & $4657 a b$ \\
\hline Procloraz & $64 \mathrm{a}$ & $124 b$ & $90.4 \mathrm{a}$ & $55.4 d$ & 3961 c \\
\hline Azoxistrobina + propiconazole & $64 \mathrm{a}$ & $127 \mathrm{a}$ & $91.3 \mathrm{a}$ & $31.7 f$ & $5152 a$ \\
\hline Testigo & $64 \mathrm{a}$ & $119 e$ & $90.5 a$ & $95.0 \mathrm{a}$ & $2013 f$ \\
\hline CV (\%) & 1.10 & 0.74 & 4.26 & 8.12 & 14.37 \\
\hline DSH (0.05) & 1.0 & 1.3 & 5.3 & 7.3 & 681.2 \\
\hline
\end{tabular}

Medias con letras iguales en las columnas no son estadísticamente diferentes (Tukey, P $\leq 0.05)$. DF: días a floración, DM: días a madurez, AP. altura de planta, AFD: área foliar dañada, RG: rendimiento de grano, CV: coeficiente de variación, DSH: diferencia significativa honesta.

El análisis de correlación de los rasgos agronómicos, rendimiento y área foliar dañada se muestra en el Cuadro 4. Se realizó un análisis combinado de las tres localidades y de forma individual. En el análisis combinado se encontró una correlación positiva entre DF y DM. En un estudio en donde se evaluó el efecto de $S$. tritici sobre el rendimiento de trigo, Rodríguez-Contreras et al. (2008) encontraron también una correlación positiva altamente significativa entre días a floración y días a madurez. En este experimento, la correlación en las tres localidades indicó que el RG fue afectado de forma negativa por AFD (coeficientes de correlación de -0.820 a -0.880), ya que a mayor porcentaje de AFD las plantas presentaron un menor rendimiento (Cuadro 3).

Sylvester y Kleczewski (2018) también encontraron una correlación negativa (-0.77) entre el rendimiento de grano y el porcentaje de severidad de enfermedades foliares causadas por diferentes hongos en trigo. En los análisis por localidad, en los tres casos se observó correlación positiva entre DM y RG, ésto se explica por el efecto que tuvieron algunos fungicidas sobre el retraso de la senescencia de la planta. Zhang et al. (2010) y Brinkman et al. (2014) mencionan que el retraso de la senescencia, determinado con base en la pérdida del contenido de clorofila y proteína soluble en las hojas, permitió que la planta produjera mayor cantidad de asimilados, mismos que fueron aprovechados por el grano.
En este análisis, en los tres casos se encontró correlación negativa entre DM y AFD, lo que indica que conforme las plantas estuvieron más días en campo con follaje verde, los hongos estuvieron más tiempo afectándolas, tuvieron mayor oportunidad de causar infección, y por lo tanto, de afectar más área foliar. Así mismo, se encontró correlación negativa en este estudio entre RG y AFD, lo que indicó que a mayor severidad de las enfermedades el rendimiento fue menor y se corrobora con el análisis de regresión entre estas dos variables (Figura 2).

Al aplicar los fungicidas, se observaron niveles diferenciales de severidad de la enfermedad que dieron lugar a los correspondientes aumentos de rendimiento. Los datos se usaron para modelar la relación entre el AFD y RG mediante regresión lineal por localidad (Figura 2). De acuerdo con los resultados, se observó que el AFD estuvo altamente asociada de forma negativa con el RG con $\mathrm{R}^{2}$ de 0.67 a 0.77 en las tres localidades. Esta tendencia negativa también fue reportada por Rodríguez-Contreras et al. (2008) al analizar la relación entre el rendimiento y el daño del área foliar causado por S. tritici, y por Bhathal et al. (2003), quienes observaron también esta asociación negativa ( $R^{2}$ de 0.65 a 0.90) entre rendimiento y severidad causada por Pyrenophora tritici-repentis en trigo. Con base en el presente análisis, por cada unidad de reducción del área foliar dañada se aumentará el rendimiento de grano en $45.5 \mathrm{~kg} \mathrm{ha}^{-1}$ en la localidad de Juchitepec, en $54.7 \mathrm{~kg}$ $\mathrm{ha}^{-1}$ en Nanacamilpa y $26.20 \mathrm{~kg} \mathrm{ha}^{-1}$ en Santa Lucia. Estos 

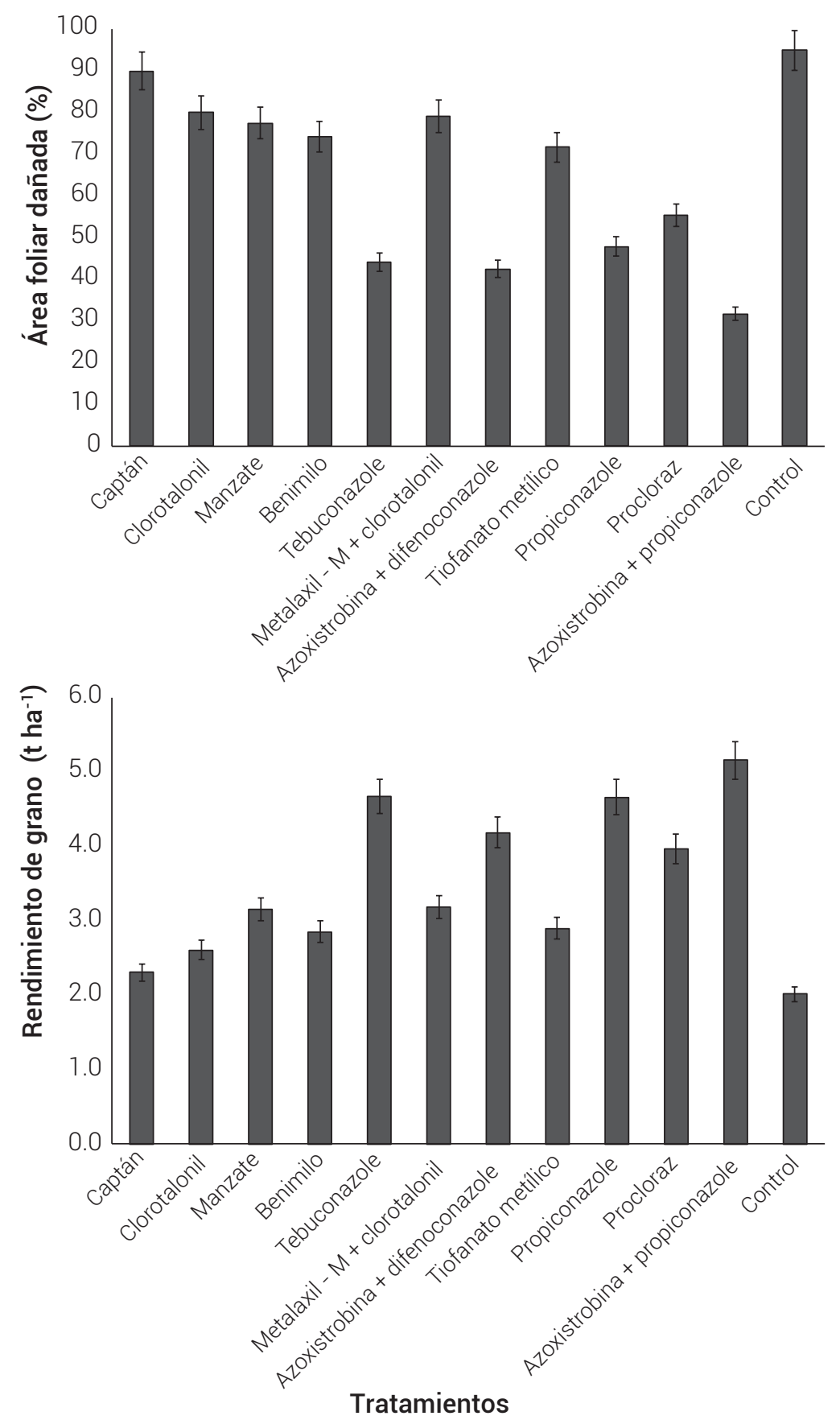

Figura 1. Porcentaje de área foliar dañada en plantas de trigo bajo diferentes tratamientos foliares y rendimiento de grano obtenido por tratamiento.

resultados muestran el beneficio potencial de la aplicación de fungicidas en el cultivo de trigo para el manejo de manchas y tizones foliares.

\section{CONCLUSIONES}

Las manchas y tizones foliares pueden causar pérdidas de rendimiento hasta del $61 \%$ en variedades susceptibles de trigo de temporal. Los ingredientes activos azoxistrobina + propiconazole causan un retraso sobre los días a madurez de la planta. Las plantas que recibieron estos mismos ingredientes activos más tebuconazole, propiconazole y azoxistrobina + difenoconazole presentaron una menor área foliar dañada y un aumento del rendimiento de grano de $8 \%$ hasta $56 \%$. Los fungicidas con azoxistrobina y de la familia de los triazoles inducen el efecto stay-green 
Cuadro 4. Coeficientes de correlación de caracteres agronómicos, rendimiento y área foliar dañada de trigo de temporal bajo diferentes tratamientos con fungicidas en tres localidades. Ciclo primavera-verano 2016.

\begin{tabular}{lccccc}
\hline Localidades & Variables & DM & AP & RG & AFD \\
\hline General & DF & $0.949 * *$ & 0.681 & 0.238 & 0.263 \\
& DM & - & 0.721 & 0.400 & 0.124 \\
Santa Lucía & AP & - & - & 0.324 & 0.292 \\
& RG & - & - & - & -0.561 \\
& DF & 0.048 & -0.039 & 0.028 & 0.013 \\
& DM & - & -0.012 & $0.856 * *$ & -0.829 ** \\
Juchitepec & AP & - & - & -0.161 & 0.264 \\
& RG & - & - & - & -0.841 ** \\
& DF & -0.161 & 0.047 & -0.028 & 0.208 \\
Nanacamilpa & DM & - & 0.076 & $0.750 * *$ & $-0.804 * *$ \\
& AP & - & - & 0.003 & 0.114 \\
& RG & - & - & - & -0.820 ** \\
& DF & 0.175 & -0.030 & 0.158 & -0.246 \\
& DM & - & 0.086 & $0.814 * *$ & -0.872 ** \\
& AP & - & - & 0.091 & -0.014 \\
& RG & - & - & - & $-0.880 * *$ \\
\hline
\end{tabular}

DF: días a floración, DM: días a madurez, AP. altura de planta, AFD: área foliar dañada, RG: rendimiento de grano; **: significancia (P $\leq 0.01)$.

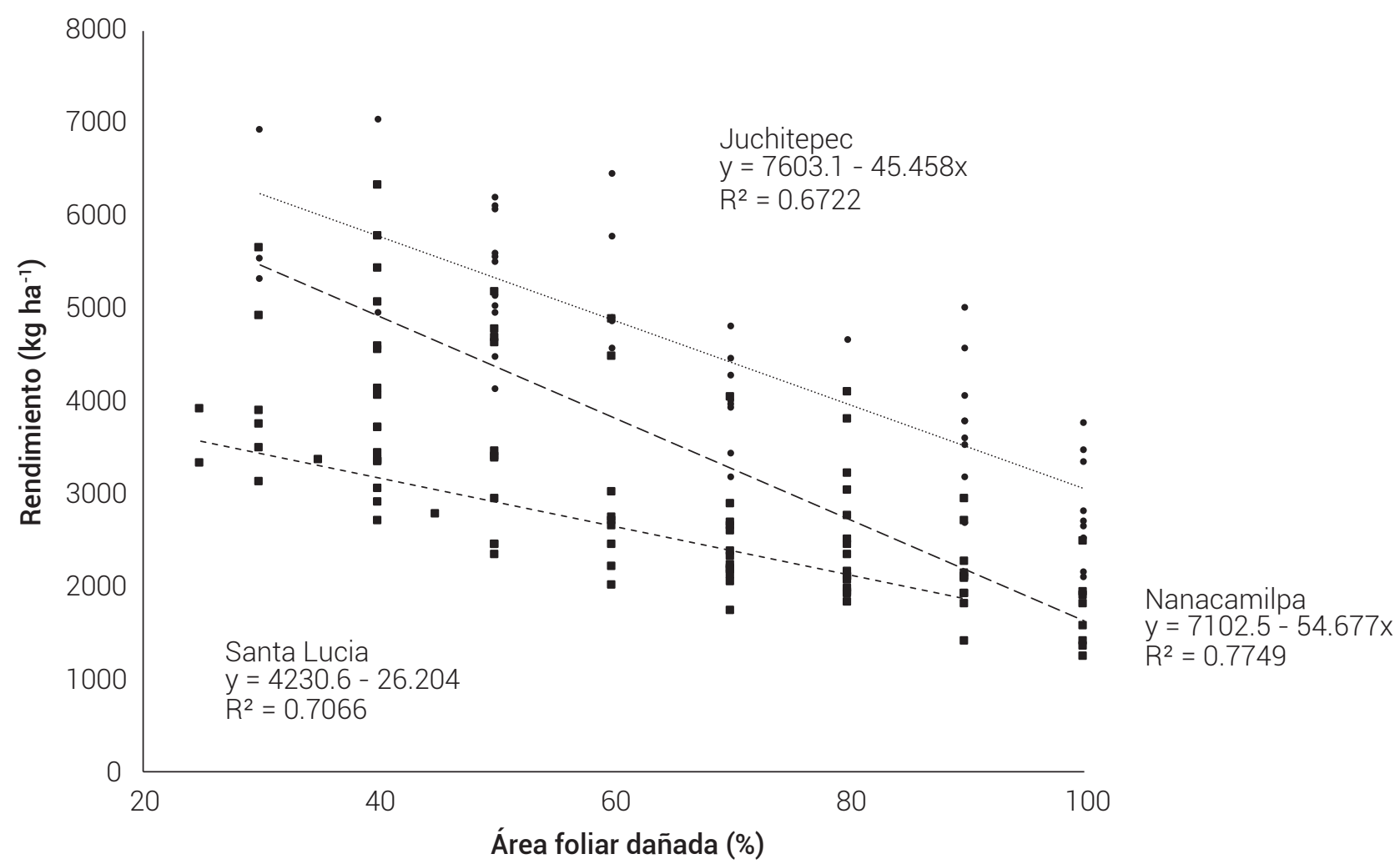

Figura 2. Análisis de regresión entre las variables rendimiento de grano y área foliar dañada en trigo de temporal por localidad. Ciclo primavera-verano 2016. 
en las plantas y provocan retraso en la senescencia. La protección proporcionada por algunos fungicidas retrasó la senescencia de las plantas e indujo un incremento en el rendimiento.

\section{BIBLIOGRAFÍA}

Acharya K., A. K. Dutta and P. Pradhan (2011) Bipolaris sorokiniana (Sacc.) Shoem.: the most destructive wheat fungal pathogen in the warmer areas. Australian Journal of Crop Science 5:1064-1071.

Aung K., Y. Jiang and S. Yang He (2018) The role of water in plantmicrobe interactions. The Plant Journal 93:771-780, https:// doi.org/10.1111/tpj.13795

Bautista E. M. E., S. G. Leyva M., H. E. Villaseñor M., J. Huerta E. y L. A. Mariscal A. (2011) Hongos asociados al grano de trigo sembrado en áreas del centro de México. Revista Mexicana de Fitopatología 29:175-177.

Beck C., E. C. Oerke and H. W. Dehne (2002) Impact of strobilurins on physiology and yield formation of wheat. Mededelingen (Rijksuniversiteit te Gent. Fakulteit van de Landbouwkundige en Toegepaste Biologische Wetenschappen) 67:181-187.

Bhathal J. S., R. Loughman and J. Speijers (2003) Yield reduction in wheat in relation to leaf disease from yellow (tan) spot and Septoria nodorum Blotch. European Journal of Plant Pathology 109:435443, https://doi.org/10.1023/A:1024277420773

Brinkman J. M. P., W. Deen, J. D. Lauzon and D. C. Hooker (2014) Synergism of nitrogen rate and foliar fungicides in soft red winter wheat. Agronomy Journal 106:491-510. https://doi.org/10.2134/ agronj2013.0395

Castro A. C., M. C. Fleitas, M. Schierenbeck, G. S. Gerard and M. R. Simón (2018) Evaluation of different fungicides and nitrogen rates on grain yield and bread-making quality in wheat affected by Septoria tritici blotch and yellow spot. Journal of Cereal Science 83:49-57, https://doi.org/10.1016/j.jcs.2018.07.014

CONAGUA, Comisión Nacional del Agua (2019) Normales Climatológicas por Estado. Servicio Meteorológico Nacional, Comisión Nacional del Agua. Ciudad de México. https://smn.conagua. gob.mx/es/informacion-climatologica-por-estado?estado= (Junio 2019)

Díaz C. H. L., S. G. Leyva M., H. E. Villaseñor M., M. Vargas H., R. Hortelano S. R., Y. R. Valdez R. y E. Martínez C. (2018) Control químico de la roya lineal en diferentes etapas de desarrollo del trigo en Terrenate, Tlaxcala. Revista Mexicana de Ciencias Agrícolas 9:1067-1074, https://doi.org/10.29312/remexca.v9i5.1510

Duveiller E., R. C. Sharma, D. Mercado, H. Maraite, M. R. Bhatta, G. OrtizFerrara and D. Sharma (2005) Controlling foliar blight of wheat in south Asia: a holistic approach. Turkish Journal of Agriculture and Forestry 29:129-135.

Duveiller E., R. P. Singh and J. M. Nicol (2007) The challenges of maintaining wheat productivity: pests, diseases, and potential epidemics. Euphytica 157:417-430, https://doi.org/10.1007/ s10681-007-9380-z

Gill K. K., R. Babuta, G. S. Buttar and P. K. Kingra (2015) Impact of climate change on wheat productivity in Ludhiana and Bathinda of Punjab. Indian Journal of Agricultural Research 49:368-372, https://doi.org/10.5958/0976-058X.2015.00067.0

Hortelano S. R. R., H. E. Villaseñor M., E. Martínez C., M. F. Rodríguez G., E. Espitia R. y L. A. Mariscal A. (2013) Estabilidad de variedades de trigo recomendadas para siembras de temporal en los Valles Altos de la Mesa Central. Revista Mexicana de Ciencias Agrícolas 4:713-725, https://doi.org/10.29312/remexca. v4i5. 1170

INEGI, Instituto Nacional de Estadística y Geografía (2019) Conjunto de datos vectoriales escala 1:1 000 000. Unidades climáticas. Instituto Nacional de Estadística y Geografía. Aguascalientes, México. https://www.inegi.org.mx/app/biblioteca/ficha. html?upc $=702825267568$ (Junio 2019).

Leslie J. F. and B. A. Summerell (2006) The Fusarium Laboratory Manual.
Blackwell Publishing. Ames, lowa, USA. 388 p

Mariscal A. L. A. (2014) Enfermedades. In: Tecnología para la Producción Sustentable de Trigo de Riego en el Bajío. E. Solís M. y A Guillén G. (comps.). Libro Técnico No. 6. INIFAP-CEBAJ, Celaya, Guanajuato, México. pp:166-186.

Mariscal-Amaro L. A., E. Solís-Moya, S. G. Leyva-Mir, J. L. Anaya-López y H. E. Villaseñor-Mir (2017) Micoflora asociada a manchas y tizones foliares en trigo (Triticum aestivum L.) de riego en El Bajío, México. Agrociencia 51:189-200.

Noriega-Carmona M. A., F. Cervantes-Ortiz, E. Solís-Moya, E. AndrioEnríquez, J. A. Rangel-Lucio, G. Rodríguez-Pérez, M. Mendoza-Elos y J. G. García-Rodríguez (2019) Efecto de la fecha de siembra sobre la calidad de semilla de trigo en el Bajío, México. Revista Fitotecnia Mexicana 42:375-384, https://doi.org/10.35196/ rfm.2019.4.375-384

Orantes-García C., E. R. Garrido-Ramírez, N. Espinoza-Paz y R. QuirogaMadrigal (2011) Resistencia de varios genotipos de trigo (Triticum aestivum L.) a Fusarium graminearum Schwabe cultivados en Chiapas, México. Tropical and Subtropical Agroecosystems 14:209-220.

PLM Editores (2011) Diccionario de Especialidades Agroquímicas. $21^{\text {a }}$ edición. PLM México S.A. de C.V. México, D. F. 1521 p.

Rattu A. R., S. Asad, M. Fayyaz, M. Zakria, S. Iftikhar and Y. Ahmad (2011) Status of foliar diseases of wheat in Punjab, Pakistan. Mycopath 9:39-42.

Rodríguez-Contreras M. E., H. E. Villaseñor-Mir, G. Leyva-Mir, J. HuertaEspino, J. S. Sandoval-Islas y H. M. de los Santos-Posadas (2008) Efecto de Septoria tritici en el rendimiento de trigo de temporal en ambientes lluviosos de los Valles Altos Centrales de México. Agrociencia 42:435-442.

Saari E. E. and J. M. Prescott (1975) A scale for appraising the foliar intensity of wheat diseases. Plant Disease Reporter 59:377380

SAS Institute (2011) SAS/STAT® 9.3 User's Guide. SAS Institute Inc. Cary, North Carolina, USA 8621 p.

SIAP, Servicio de Información Agroalimentaria y Pesquera (2019) Cierre de la producción agrícola por estado y cultivo. Servicio de Información Agroalimentaria y Pesquera. SADER. Ciudad de México. http://www.siap.gob.mx/cierreagricola/ (Febrero 2019).

Sylvester P. N. and M. N. Kleczewski (2018) Evaluation of foliar fungicide programs in mid-Atlantic winter wheat production systems. Crop Protection 103:103-110. https://doi.org/10.1016/j. cropro.2017.09.012

Villaseñor-Mir H. E., E. Espitia-Rangel, J. Huerta-Espino, E. Solís-Moya, A. María-Ramírez, D. Aguirre-Montoya and R. J. Peña-Bautista (2005) Registration of 'Nahuatl F2000' wheat. Crop Science 45:424425, https://doi.org/10.2135/cropsci2005.0424

Villaseñor M. H. E., J. Huerta E., R. Hortelano S. R., E. Martínez C., E. Espitia R., E. Solís M., L. Osorio A. y M. F. Rodríguez G. (2018) Valles F2015: nueva variedad de trigo para siembras de temporal en México. Revista Mexicana de Ciencias Agrícolas 9:1315-1320, https:// doi.org/https://doi.org/10.29312/remexca.v9i6.1592

Villaseñor M. H. E., R. Hortelano S. R., E. Martínez C., L. A. Mariscal A., S. G. Leyva M. y J. Huerta E. (2012) Control químico de las enfermedades: una alternativa para la producción de trigo de temporal en Tlaxcala. Revista Mexicana de Ciencias Agrícolas 3:595-600, https://doi.org/10.29312/remexca.v3i3.1452

Wegulo S., J. Stevens, M. Zwingman and P. S. Baenziger (2012) Yield response to foliar fungicide application in winter wheat. In: Fungicides for Plant and Animal Diseases. D. Dhanasekaran (ed.). InTech. Rijeka, Croatia. pp:227-244, https://doi.org/10.5772/25716

Zhang Y. J., X. Zhang, C. J. Chen, M. G. Zhou and H. C. Wang (2010) Effects of fungicides JS399-19, azoxystrobin, tebuconazole, and carbendazim on the physiological and biochemical indices and grain yield of winter wheat. Pesticide Biochemistry and Physiology 98:151-157, https://doi.org/10.1016/j. pestbp.2010.04.007

Zillinsky F. J. (1984) Guía para la Identificación de Enfermedades en Cereales de Grano Pequeño. CIMMYT. México, D.F. 141 p. 